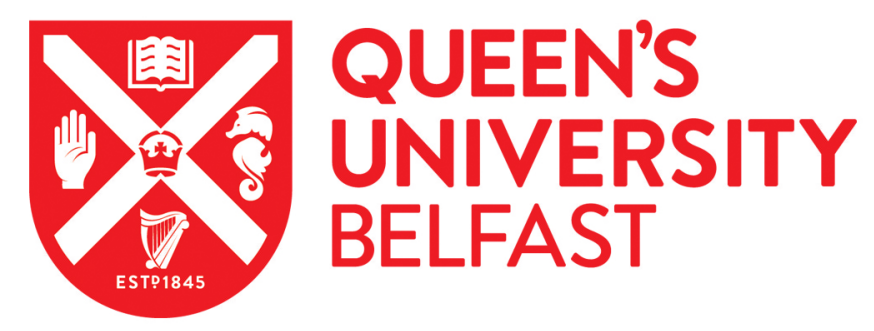

\title{
An interactive and immersive human-computer interface for rapid composite part production design
}

McConnell, R., Butterfield, J., Rafferty, K., Price, M., Murphy, A., Burke, R., Legg, A., \& Lemon, R. (2018). An
interactive and immersive human-computer interface for rapid composite part production design. Proceedings of
the Institution of Mechanical Engineers, Part B: Journal of Engineering Manufacture, 232(13), 2276-2285. https://doi.org/10.1177/0954405417703427

Published in:

Proceedings of the Institution of Mechanical Engineers, Part B: Journal of Engineering Manufacture

Document Version:

Peer reviewed version

Queen's University Belfast - Research Portal:

Link to publication record in Queen's University Belfast Research Portal

Publisher rights

Copyright IMechE 2017.

This work is made available online in accordance with the publisher's policies. Please refer to any applicable terms of use of the publisher.

\section{General rights}

Copyright for the publications made accessible via the Queen's University Belfast Research Portal is retained by the author(s) and / or other copyright owners and it is a condition of accessing these publications that users recognise and abide by the legal requirements associated with these rights.

Take down policy

The Research Portal is Queen's institutional repository that provides access to Queen's research output. Every effort has been made to ensure that content in the Research Portal does not infringe any person's rights, or applicable UK laws. If you discover content in the Research Portal that you believe breaches copyright or violates any law, please contact openaccess@qub.ac.uk. 


\title{
An Interactive and Immersive Human-Computer Interface for Rapid Composite Part Production Design
}

\author{
R. Mc Connell ${ }^{1}$, J. Butterfield ${ }^{1}$, K. Rafferty ${ }^{1}$, M. Price ${ }^{1}$, \\ A.Murphy ${ }^{1}$, R. Burke ${ }^{2}$, A. Legg $^{2}$, R. Lemon ${ }^{2}$ \\ ${ }^{1}$ Queens University Belfast, ${ }^{2}$ Bombardier Aerospace
}

\begin{abstract}
This paper addresses the need for better retention and exploitation of tacit knowledge for intelligent computer aided design. It presents an automated design framework for the development of individual part forming tools for a composite stiffener incorporating parametrically developed design geometries. The work develops existing principles in knowledge based engineering and parametric modelling beyond product design into the manufacturing planning domain. Outcomes demonstrate chronological benefits in automated process design methods as well as learning enhancements as the tacit knowledge data set can now include an applied element through an auto-generated virtual build environment. A virtual environment presenting a design concept to the planner for interactive assembly assessment was generated in 20 seconds and enabled the completion of virtual builds in support of the development of an optimal forming tool arrangement. This principle enables the addition of an experiential tacit knowledge feedback loop to further improve assembly planning for design concepts as they evolve. Challenges still exist in determining the level of reality required to provide an effective learning environment in the virtual world. Full representation of physical phenomena such as gravity, part clashes and the representation of standard build functions require further work to represent real physical phenomena robustly.
\end{abstract}

\subsection{Introduction}

The aerospace industry, like others, faces considerable market demands of ever improving performance and ever reducing costs and time scales for delivery. Significant commercial advantage can be gained by delivering the right product to the market at the right time and ahead of competitors. Such pressure congests the design and development programmes for new aircraft and risks the delivery of right-first-time innovative solutions. By reducing the time-to-market the net cost of the design process is not necessarily reduced. The time-to-market may be reduced by carrying out the same amount of work, but compressing it into a shorter schedule. This can result in higher design and manufacturing preparation costs. The goal should therefore be to reduce the flow time required to design an aircraft by reducing the hours required to perform the design and manufacturing preparation tasks.

From a commercial point of view it is recognised that competitive advantage can only be maintained through the application and foundation of knowledge. It is also recognised that in many organisations their operating knowledge is un-documented [1] with up to $90 \%$ of organisational knowledge existing within the heads of their employees in a tacit 
form. A significant proportion of this can be regarded as high value intellectual property which is unique to the individual and their employer. Knowledge is therefore a key asset just like capital equipment on the shop floor or cash in the bank. Despite its importance, knowledge in general and tacit knowledge in particular, can be at best under utilised or at worst lost [1] through outsourcing, downsizing, mergers, redundancies, retirements or the natural migration of individuals in a competitive labour marketplace. Technical knowhow and competitive advantage can be forfeited to a competitor or lost altogether as $21^{\text {st }}$ century business practices and commercial operating conditions make all of these factors commonplace in industry. Knowledge management and the maintenance of any competitive advantage remain a significant challenge and it is acknowledged that this needs to be addressed in what has become a turbulent business environment [2].

In a technical context, sustainable competitive advantage can flow from the creation, ownership, protection and use of difficult-to-imitate commercial and industrial knowledge [3]. However, despite improvements in information technology and data management, the process of knowledge generation, interpretation, exploitation and even retention, still requires human intervention. The tacit knowledge of domain experts remains central to technical and commercial success of commercial engineering programs. Digital methods such as computer aided design (CAD), finite element analysis (FEA), computational fluid dynamics (CFD) and product lifecycle management (PLM) have now matured to the extent that they have become ubiquitous across most engineering design disciplines. Multidisciplinary interaction has become routine as interface methods and codes have been developed which allow the seamless interchange of data between platforms and disciplines. These methods and software platforms have the capacity to generate the information required to develop optimal engineering solutions. Opportunities still exist to enhance and exploit automated design methods through better use of tacit design knowledge and broader use of virtual reality methods to enhance it, as a product and its manufacturing requirements evolve. If these opportunities can be exploited then OEMs would be in a better position to overcome the perennial problems of time and cost overruns on major product development programs $[4,5]$ as well as the issues arising from diminishing competitive advantage due to loss or under-exploitation of organisational knowledge.

Intelligent design systems are already in use in aerospace. 'Artificial' intelligence (AI) already utilises computational methods to emulate various faculties of human intelligence and biological metaphors [6,7]. They use a combination of symbolic and sub-symbolic systems capable of evolving human cognitive skills and intelligence and they are ideally suited for tasks such as search and optimisation, pattern recognition and matching, planning, uncertainty management, control and adaptation. However, the capabilities made available through the use of (AI) come at the cost of computational expense both in their development and application. Although they promote the evolution of knowledge there has to be a question as to whether their application can mimic and fully exploit existing knowledge efficiently. An alternative is the development of design capability where the intelligence is derived from direct capture and integration of tacit knowledge in parametrically driven tools. This subdivision of knowledge management into codification approaches that emphasise the collection and organisation of knowledge (e.g. artificial intelligence) and personalisation approaches that emphasise human resources and communication (e.g. capture and use of tacit knowledge) is advocated by McMahon 
et. al. [8]. The challenge associated with the capture of design rationale [8] is an integral part of the work presented here.

Previous work has demonstrated the benefits of automated design methods and has shown how aircraft concepts can be transformed directly from a simple 1D structural representation to full blown 3D computer aided design (CAD) model [9]. This effectively coupled the structural analysis and CAD domains using analytical outputs to drive the automated generation of detailed 3D features and geometries. The methods used effectively removed the human in the loop who would otherwise have translated analysis outcomes into physical elements for the purpose of then 'hand generating' the 3D model. The tacit knowledge of the designer (based on the functions available within the CAD user interface) was effectively captured and added to the design code in order to translate structural performance characteristics into conceptual architectures. This significantly reduced development lead times enabling shortened detailed design phases or the opportunity to explore more options with equivalent development lead times thereby resulting in more optimal outcomes.

Methods have also been developed to design aircraft panel assembly fixtures automatically based on rules derived from the geometric properties of the product itself [10]. The product dimensions arising from the structural design process [9] were used as the basis for the design of the equipment required to assemble panel sections accurately. In this case the tacit knowledge of the tooling designer was included in the process and included strategies for the sub-division of large fuselage sections into smaller sections for the manufacture and assembly of individual panels as well as the use of standard sections and clamping arrangements for the fixture itself. This then coupled the design and assembly domains, facilitating the design of fixtures, work cells and even factory layouts which could evolve in parallel with the product. This again offered opportunities for reducing development lead times by considering assemblability earlier in the product development cycle.

In both cases [9 \& 10] the automatic generation of CAD geometry (for the product and its assembly fixtures) was built on data related to physical sizing derived from structural performance. The engineering principles surrounding structural analysis as well as standard practices in fixture design for metallic assembly processes are relatively well established. Their inclusion in automated design tools was therefore relatively straight forward. However, as new technologies develop to support the application of novel material systems in aircraft design, the ability to create support equipment becomes less straight forward. Domain and even material specific knowledge still requires the input of subject experts making the capture and use of this tacit knowledge critical to the development of automated tools for specialised manufacturing applications. One such application is the design of composite forming tools.

Although by definition, automated methods require tacit knowledge for their definition in the first instance the creation of design concepts is only the first step in a process that leads to its eventual manufacture. The exploitation of tacit knowledge for assembly based tasks around new products, can only be maximised when the operator completes the task and this is not serviced by current capabilities in automated design. To address this, this work focuses on the development of an automated design framework for the development of tools suitable for forming a carbon fibre reinforced stiffener. The 
framework uses parametrically developed design geometries for both the part and its layup tool. The framework has been developed with a functioning user interface where part / tool combinations are developed using the interdependent dimensional relationships between the 'as designed' composite part and the tool required to form it. The tacit knowledge or 'know how' of the tooling engineer has also been coded into the tool covering design elements away from the part / tool interface. When complete the design options can be passed to a virtual environment for utility based assessment of their features and assemblability characteristics. This enables the integration of established, design based tacit knowledge with an additional experiential element made possible through the completion of virtual builds. In the context of this paper, automated design is defined as: 'the generation of 3D CAD geometry automatically, through a custom coded user interface, without direct user interaction with the traditional CAD interface'. Similar components differing only on key dimensions are used for the work. Part details, manufacturing procedures and tooling features are captured and embodied in the automated design code. The approach includes the use of the required geometrical relationships between the tool and part as well as the peripheral design rules required to form the tool geometry beyond the part / tool contact surfaces. These are used in turn to generate and articulate design options. The work includes the transfer of the resulting component and tool geometry to a VR environment enabling the virtual assessment of tooling functions as advocated by McConnell et. al. [10]. By providing an interactive and immersive human-computer interface this work creates an efficient framework for designing, planning and assessing composite part manufacture including tooling functionality with respect to the human user. The outcomes enable the direct inclusion of what was previously considered artisan or 'black art' type manufacturing knowledge in engineering design and product build assessment.

\subsection{Method}

\subsection{Automated Design Framework}

Figure 1 maps the framework used for the development of the composite layup tool required to form the stiffener. The process includes component inspection and definition of the data required to drive mould tool design. This is then used to develop tool forms based on critical features and dimensions as well as 'in house' design rules (i.e. draft angles, standard fasteners etc.) and mould tool functions.

\section{Component: $\quad$ Mould Design: $\quad$ Virtual Reality: \\ - Inspection \\ - Design \\ Data \\ - Design Automation \\ - Design Rules \& Equations \\ - Inspection \\ - Assembly / \\ Disassembly}

Fig. 1. Automated Design Framework for Virtual

Development of Composite Layup Tool. 
These include the elements required for ease of handling by the user and how the tool integrates with the curing environment (i.e. dimensions appropriate for insertion and positioning in autoclave as well as positioning on loading / unloading stations). The stiffener and its associated mould tool are then transferred to the virtual environment for functional assessment where a user can interact with all elements of the system.

\subsection{Structural Stiffener}

Fig 2 shows the basic stiffener with the main dimensions highlighted. This component currently exists in two forms and the dimensions highlighted in Fig 2 vary for each case. Both versions of the stiffener have the same Thickness, Length, Width 1 and Width 2. All other dimensions differ for the two variants. The aim of this work was to create a third variant of the stiffener based on the design rules and key dimensions derived from the existing components. During the inspection phase a subtle change in the profile shape along the surface defined by Widths $2 \& 3$ and Depths $2 \& 3$ was identified. A decreasing gradient along this surface meant that there was a variation between the two stiffener versions as the parts have to fit in with different structural profiles during final assembly.

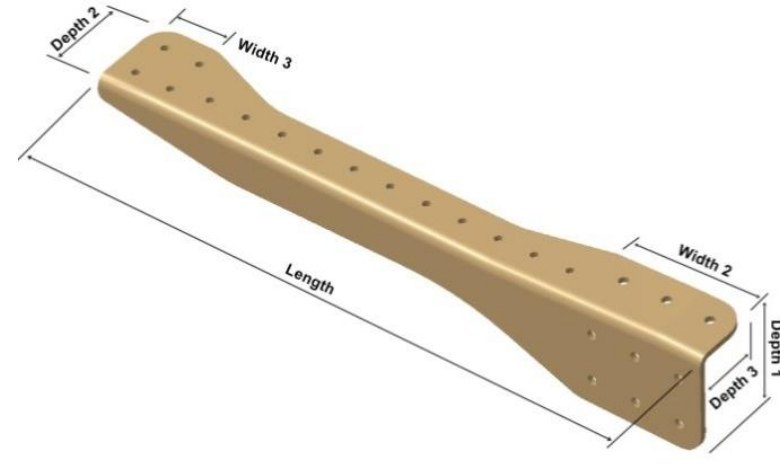

Fig. 2. Composite Stiffener.

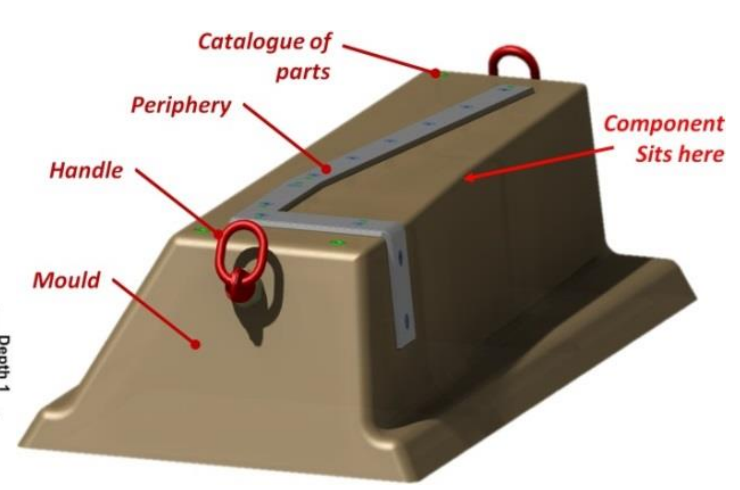

Fig. 3. Composite Layup Tool.

\subsection{Layup Tool}

Fig 3 shows the composite layup tool with the main features highlighted, these are: The Mould Block which is a shelled feature with two underlying ribs, three holes required on upper surface as well as a further eighteen holes for the periphery bar.

The Periphery Bar - which consists of three separate components and a total of eighteen holes required for fixing the component. Two Handles on the ends of the block and the Catalogue of Parts (Nuts, bolts and screws). The process for automated mould design started with the assessment of the features on the existing mould tools. This identified key features and dimensions. The moulds include multiple holes which vary in size however, despite the dimensional differences between the parts and mould for the two existing stiffeners, the hole properties on the tools remain identical for both.

The tool inspection process also included the comparison of the hole positions and part locations relative to a datum point located at the corner of the upper tool surface. See 
Figure 4. The mould tool also reflected the decreasing gradient along top surface of the stiffener. This feature is an important aspect of the layup tool as it forms a critical feature which interfaces with a higher profile on the aircraft during final assembly. The automated development of this feature is critical to the overall success of the work as it influences assembly accuracy.

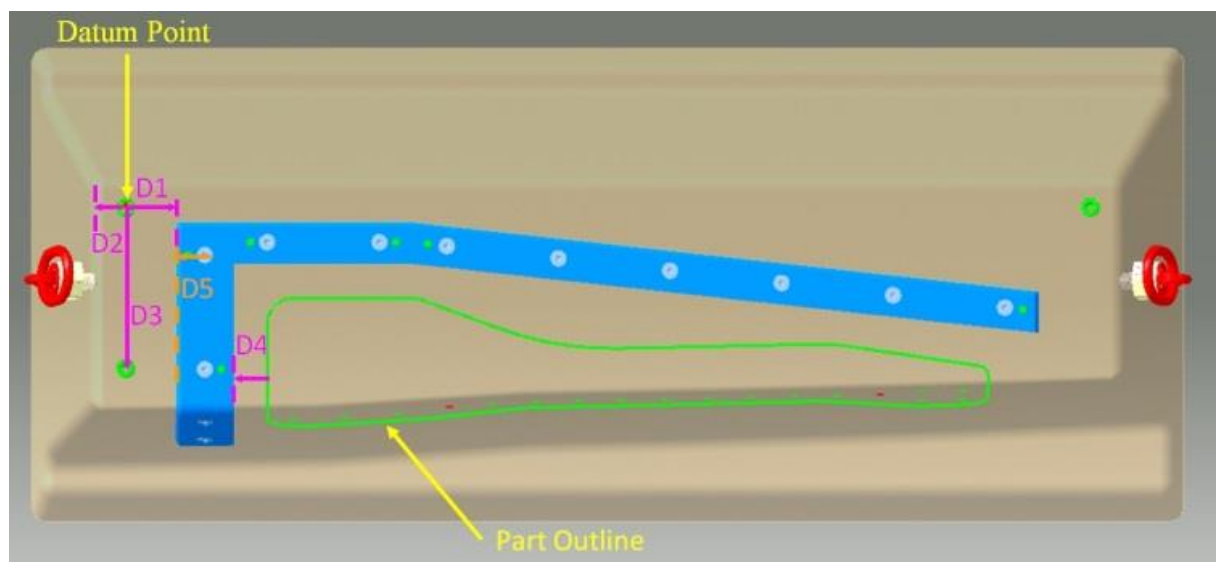

Fig. 4. Tool Inspection process: Definition of Positional

Parameters for Automated Design Process.

\subsection{Computer Aided Design}

Relating physical features to the CAD functions required to model them, was a key element of this work. The key features on the tool were the part forming surfaces on the mould and the two end mounted handles. See Figure 3. Each of the primary physical features was sub-divided into the constituent parts (see Figure 5) and the next stage in the process required the translation of this feature data using a process that would enable the creation of the CAD models including the spatial relationships between features and parts. Once placed in the CAD environment the creation of repeated parts and features involved feature generation and copying functions. Axis systems were defined and used to control orientations and relative positions. This process formed the basis for a procedure which could automate the design of further components and tools differing only in key dimensions. Figure 6 maps the geometry creation process in the form of a design tree.

Within the CAD environment the automated application of the modelling functions meant that some of the design components could be transferred from a parent part. This removed access to their design parameters and the system considered these as elements within a catalogue of standard parts. Although this meant that detailed design knowledge for these parts was limited, mapping out names and matching features was helpful when automating the design later in the process. This method was applied to the mould handles as well as the periphery bars. With all of the required design data gathered for both the stiffener and the mould tool, the process of automating the generation of further components could begin. 


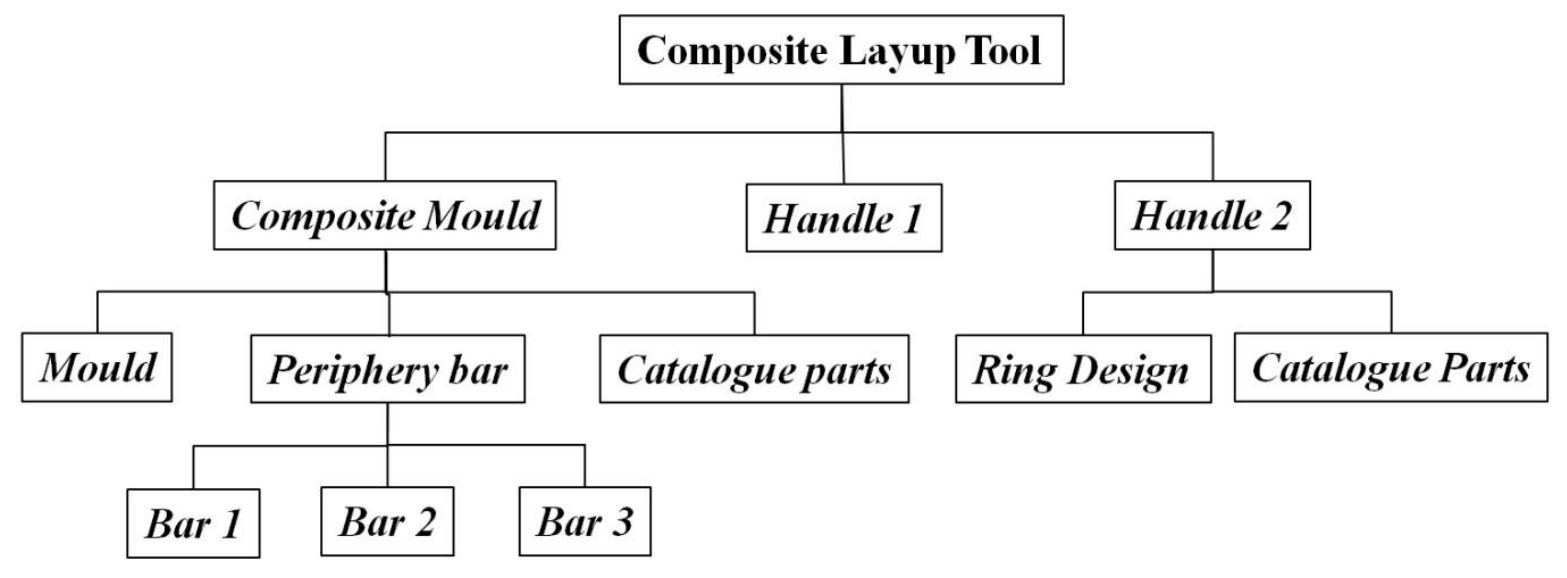

Fig. 5. Primary Geometric Elements for Composite Layup Tool.

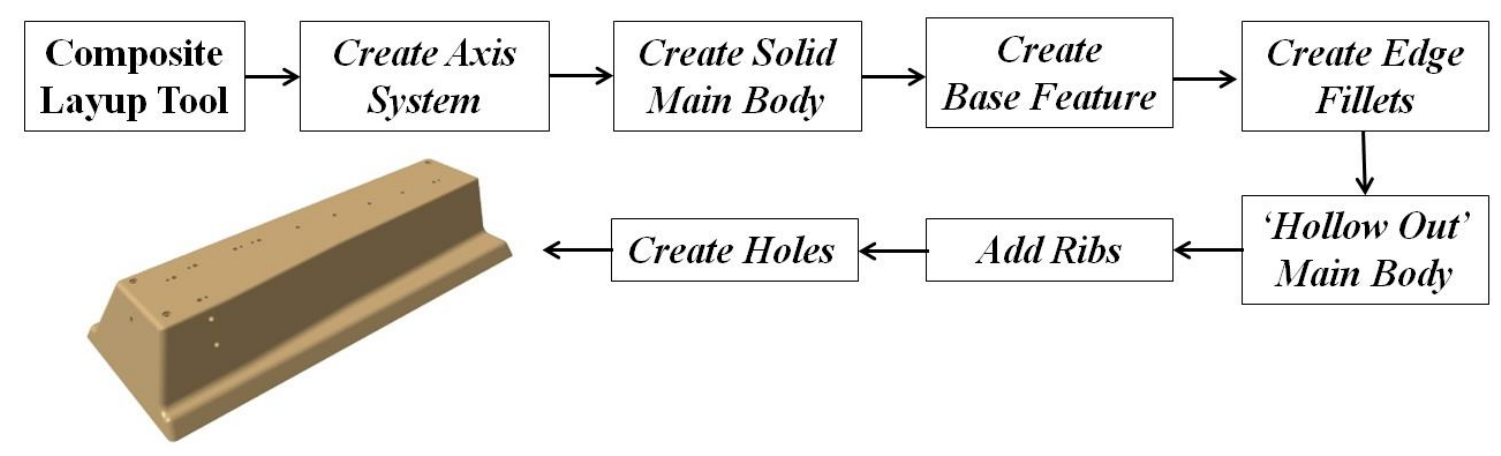

Fig. 6. Design Tree for Composite Layup Tool.

\subsection{Automated Design}

A user interface had previously been developed for the automated design of complex metallic assemblies [9]. This environment was re-used for this work (see Figure 7) and functionality was extended to include the design of the composite layup tool.

The interface was used only for the input of critical dimensions as required design variables. All knowledge, rules and mathematical functions were embodied and stored within the write protected VBA script. Lower level design parameters that were derived from the higher level component properties were also stored and accessed via the VBA script. An example was the size of the individual periphery bars which were based on the dimensions of the mould tool which was in turn, based on the size of the part. This predefined data structure helped to ensure the dimensional integrity of the components. If any changes were made to the high level component, the low level parts were updated automatically, maintaining the design intent at all levels from the component through to the tool required to lay up and form it.

The user form can be broken down into three sections; non-design inputs, part design inputs \& tool design inputs. The information on the forms was kept to a minimum and the user had just enough control to design the required component variant. Inputs required 
for repeated features came through the code - inputs required for the variable features came from the user via the interface. In addition to core design functions the interface included file management capability for accessing, naming and saving documents and CAD files.

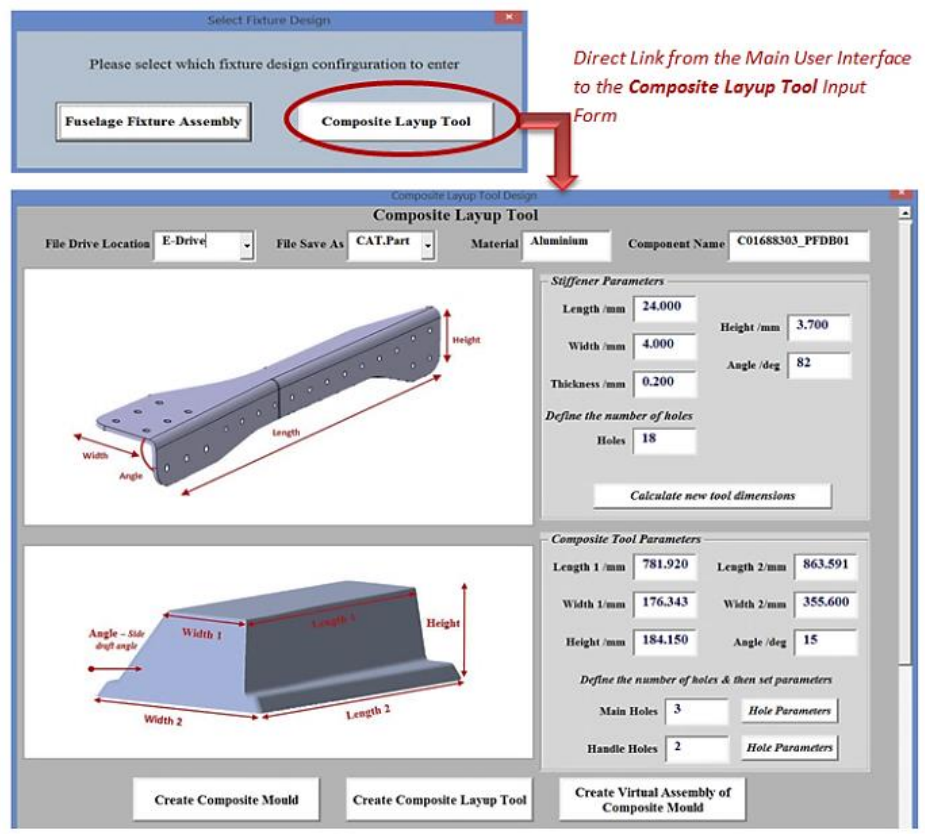

Fig. 7. User Interface For Automated Design of Composite Layup Tool For Aerospace Stiffener.

From the input of the stiffener parameters, the system could calculate the size of the composite layup tool based on the geometric information established during the analysis phases. These were subsequently combined with standard design practices and converted to the series of design rules used to derive the final tool geometry.

\subsection{Virtual Reality}

The virtual world is constructed using Python script for the VR software Vizard which can provide a fully immersive experience in a virtual environment. Unlike a typical CAD interface were geometry creation and manipulation functions are presented to the user in multi layered toolbars, VR platforms typically do not have a default user interface. All operations required when the user is in the environment must be coded to provide the user with the required functions when they are immersed. To achieve a realistic experience interaction was based on the movement of the user which was in turn based on the motion of their body and their inputs via a control device (A Nintendo Wii remote in this case). The user needed to visualise and interact with the immersive environment. Immersion in and control of the VR environment was achieved using a head mounted display (for stereoscopic visual immersion) and a motion tracking system which enabled realistic body movement as sensors attached to the head and hand in this case, were tracked within a magnetic field (see Figure 8). 
The main aim of the VR system in the context of this work, was to create a realistic space that provided functionality for completing virtual part builds. The environment used here was kept as simple as possible, and included a ground for walking on and a standard sky background. Two tables were used, one for placing containers on (with nuts, screws and bolts), and the other that accommodated the composite mould tool and the separated periphery bars. All of the components within the VR environment were taken from the CAD design concept developed using the automated design process described in earlier sections. All components were automatically dis-assembled into a starting position in the virtual world so that the user could then assemble the mould tool.

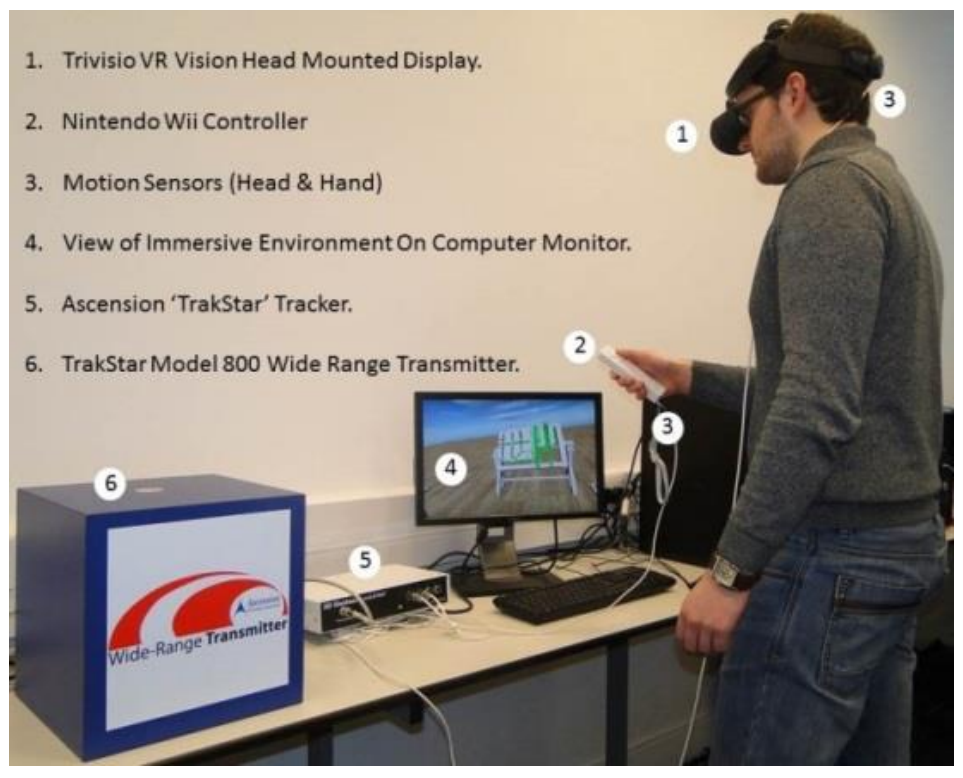

Fig. 8. Desktop Virtual Reality (VR) System.

\subsection{Results}

\subsection{Creation of Composite Mould Tool Components}

The code sequence for the creation of the composite layup tool in CATIA embodied information related to the dimensional requirements of the tool (based on the stiffener sizes), the tool configuration (for features not in direct contact with the stiffener) and the basic CAD functions required to build the geometry.

The actual VBA code for the creation of an appropriate axis system is listed in Figure 9. Equivalent functions were created for the other listed code elements which covered in sequence, all of the actions required to build the tool. A solid mould block for the main body was created first by extruding the required section. This was initially 'oversized' to allow material reduction as required to impart the required surface profiles on the mould tool which reflect the stiffener dimensions. It should be noted at this stage that the surface profiles used here were derived from existing tool geometries. These were manufactured taking into account material behaviours such as 'spring back'. The base plate was then added to the mould followed by the corner fillets. The geometry for these was governed by established design rules relating radius to material thickness. 
Set PartDocument1 = CATIA.ActiveDocument

Set Part1 = PartDocument1.part

SetaxisSystems 1 = Part1.AxisSystems

SetaxisSystem $1=$ axisSystems 1. Add ()

axissystem1.Name = "Absolute Axis"

axissystem1.IsCurrent $=0$

axissystem1.Type $=0$

'Set origin co-ordinates for axis system'

axissystem1.OriginType $=1$

$\operatorname{origincoord}(0)=0$

$\operatorname{origincoord}(1)=0$

origincoord $(2)=0$

axissystem1.PutOrigin origincoord

'Update axis system'

Part1.UpdateObject axissystem1
'Activates CATIA document'

'Add new axis system'

'Name new axis system'

'Axis system is not current'

'Axis system type set to standard'

'Origin type is defined by

co-ordinates'

Fig. 9. Code for 'Create Datum Tool Datum Axis' Function.

When the mould was formed as a solid block the 'shell' function was used to hollow it out to a standard thickness. Again this thickness was governed by standard practice. Once the mould was shelled, two ribs were added to bridge the gap between the two long sides. These were required to add dimensional stability to the mould. The rib profile varied in accordance with sectional changes along the length of the mould but the process for creating both ribs was the same. The creation of hole positions to accommodate fasteners followed the required pattern relative to the model datum (see Figure 4). Individual hole sizes were specified according to the fastener type identified during the inspection stage. Smaller tooling elements including the periphery bar handles and individual fasteners were coded in a similar way to the main body of the mould. Again, each was saved as a CatPart file in preparation for final assembly as a CATproduct. The final coded element required for this process required the presentation of the user with save options. File extensions and locations were defined by the user through the user interface.

\subsection{Assembly of Composite Mould Tool Components}

The final stage in completing the composite layup tool involved the creation of a CATIA assembly (CATproduct) and the importation of the respective components (CATparts). 
All of the individual parts were required for this stage and the system only needed to read in their final position to create the final tool assembly.

The process for opening and saving a CATIA Product document is identical to that used during the creation of the individual mould pieces, however in coding terms, instead of 'Add.("Part")' the system adds a product, 'Add.("Product")'. The challenge in this area of the system was in how the parts were added and fitted together to form the final product. Parts must be constrained and fixed relative to one another in order to create a valid assembly within CATIA. In this case the mould is the primary component within the assembly and the additional parts were positioned relative to it using $(\mathrm{x}, \mathrm{y}, \mathrm{z})$ positional and rotational coordinates.

\subsection{Assembly Analysis Using Virtual Reality (VR)}

The first step required in the construction of a virtual space for assembly analysis was to convert the CAD files to the wrl format required for the virtual world within VIZARD. This was offered as a file save option in the user interface of the automated design tool. Figure 10 shows the composite layup tool after the CAD data was migrated to the virtual environment. The 'GrabHand' script also shown in Figure 11, was used for grabbing objects with physical effects included (i.e. gravity \& component clash). In addition to the 'geometry import' and 'position set' functions, physics and grabbing functions were also included, in the virtual environmental settings.

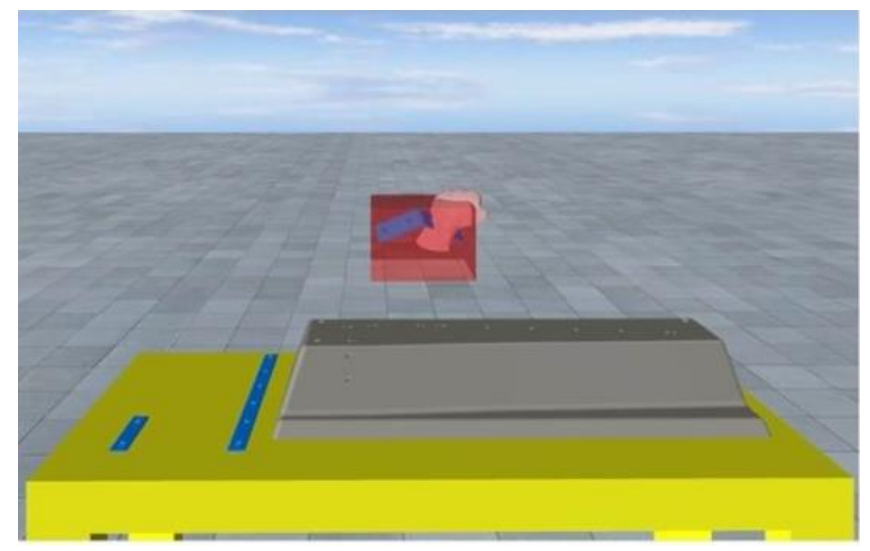

Fig. 10. Composite Layup Tool after the CAD Data Was Migrated to the Virtual Environment.

GrabObjects $=[$ Bar1, bar2, Bar3] \# List of Objects to Grab

\# Pass in the list of hands to physics engine for processing

Import GrabHand

GrabHand.GrabHandList (grabObjects, handlist=viztracker. getHandList( $)$, springs=True)

Fig. 11. Python Script For 'Grab \& Move' Function Within Vizard VR Environment. 
The grab function was clearly required to enable the user to lift and place objects in the assembly. The physics function was required to improve the sense of reality within the system where parts clash if they come in to contact and pieces fall due to the forces of gravity if they are dropped. To achieve this, collision shapes were added to all of the objects in the environment. An additional sound function helped the user to understand when a collision had occurred.

With the above functions established the user was then free to lift and place individual pieces into their 'as designed' positions. This enabled the completion of an assembly based, virtual assessment of the mould design concept where the automated tools compressed the time from concept development to virtual assembly from days / weeks to a matter of minutes. The process produced a CAD design for the composite mould tool in less than 3 minutes and created a virtual environment to interact with the design in 20 seconds.

\subsection{Discussion}

With competitive advantage and efficient knowledge management in mind, the primary aim of this work was to link an automated design framework with a virtual reality environment. The intention was to create capability which allowed the user to carry out design/assembly analysis with shorter lead times using automatic design and virtual assembly tools that allowed the production of better quality systems and products as the manufacturing systems are designed concurrently with the products. The knowledge acquired from the $\mathrm{CAD}$ documents when combined with the tacit knowledge of the tool designer, has been utilised to assemble a set of automated codes for the purpose of developing conceptual tool designs in the virtual environment. The virtual assembly function allowed the user to include tacit knowledge based on what the tooling designer contributed through the design process and what they could add based on their domain specific knowledge through the completion of virtual builds.

The work was able to successfully create an automated design process to develop a composite layup tool based on the design of the part that it will form (structural stiffener). It also showed how a direct link with the virtual world can be created to inspect, assemble and disassemble the new design concept. The process was able to produce a CAD design in less than 3 minutes and create a virtual environment with that design in 20 seconds. The approach presented in this paper will have maximum impact when used for the development of families of parts, with common features because the time required to generate the automated code will only be offset if a single, core code can be applied repeatedly. If the development process follows basic principles associated with best practice in design for manufacture and assembly (DFMA) then the lead time benefits demonstrated by this work are eminently possible. Although the work focused on a composite layup tool as single design application, the outcomes are equally applicable to any structural element manufactured using a forming tool.

Concurrent engineering (CE) systems and practices have focused on the integration and alignment between design and manufacturing; this has already proven to reduce manufacturing costs and improve product quality [11]. The use of CE in the redesign of the Airbus A340 resulted in an estimated reduction of $25 \%$ in development time and $30 \%$ product costs [12]. The success of any integrated design environment is dependent on 
individuals sharing information and collaborating during the decision making process. True concurrency is difficult to achieve. For the work completed for this paper the product geometry must exist before the tool required to form it can be considered. However, the system presented here begins to approach concurrency when the process from part concept through tool design to virtual build validation is reduced to minutes rather than days. The consideration of multiple options through iterative loops in very short time periods means that tool development is for all intents and purposes concurrent.

The learning and innovative capability of an organisation is critically dependent on its capacity to mobilise tacit knowledge and foster its interaction with explicit knowledge [13]. Establishing a framework to organise and capture this knowledge such as the one presented here, will not only preserve the knowledge it also avoids any associated loss or waste and means that it can be used repeatedly and consistently. Accurate knowledge presentation and visualisation also enables organisations to explore knowledge spaces so as to gain better understanding and insight [14].

Researchers have suggested that virtual environments could provide advantages for conceptual learning by allowing opportunities for learners to view information within the context of meaningful locations [15]. This work provides a means by which this can be achieved even at the conceptual development stage as the automated tools can place the operator in a virtual environment to gain familiarity with the manufacturing setup before prototypes or physical systems are produced. Organisational learning involves gaining experience with products and processes, achieving greater efficiency through automation and other capital investments, and making other improvements in administrative methods or personnel. Unfortunately, the core dilemma that confronts all organisations is that they learn best from experience but they never directly experience the consequences of many of their most important decisions [16]. VR capability provides a means by which the experiential elements of the learning process can now be gained before production begins. When coupled with automated design capability, the time required to create and use the VR environment is offset by significantly reduced design lead times. The time (and therefore cost) based benefits of the approach presented here are supplemented by the potential for achieving 'right first time' production systems that have been validated in the virtual world. The improved learning environment will also benefit operator learning reducing the likelihood of errors when production begins. This has been observed by other authors including Mujber [17].

Previous authors have looked at immersive virtual reality as a means of supporting process development in the manufacturing domain. Peng et. al. used VR as a means of integrating product review and production evaluation activities[18]. Although this work demonstrated the benefits of improved interaction, visualisation and resource sharing, it still required completed inputs from the design domain in terms of the geometry which populated the virtual environment. This means that design iterations would be time consuming as they require activity outside the developed system. Manesh and Hashemipour [19] as well as Korves and Loftus [20] extended VR applications beyond the product itself to develop a methodology for the development of shop floor control systems and the planning of manufacturing work cells. In both cases, their approach was 'post-design' in terms of the chronology of their application. There was therefore a lack of connectivity in their methods between process planning and the mechanisms used to 
develop and articulate concepts. This means that any developmental benefits resulting from the use of VR such as the time savings in process design and general learning benefits through improved human / environment interaction, could be offset by protracted design cycles. Pai et. al. [21] developed an augmented reality based robotic work cell to simulate a manufacturing plant environment. Although this approach resulted in visual and functional benefits to the user it still required physical assets to deliver the virtual experience. The work presented here overcomes all of these issues through the provision and integration of automated design methods and an immersive VR environment, within a single interface, so that design options can be developed and fully evaluated without the need for physical assets beyond the VR equipment.

Future manifestations of the methods presented in this paper will require development of the core design methods to include intelligent codes. These will integrate 'as manufactured' part form predictions within the design code to avoid the need for referencing / inspecting existing hardware to understand phenomena such as spring back. Further work and investment is also required to accurately determine, capture and exploit knowledge behind the design process to an extent where the automated design methods presented here can move beyond single components to span entire systems. Although this work presents the mechanism for placing the designer in front of his/her creations in a virtual environment, further work is required to quantify the learning benefits of this approach.

By combining parametric design methods with improved exploitation of tacit knowledge, the potential to improve the design system generally, produce better quality products with more efficient manufacturing systems and reduced lead times, becomes a reality.

\subsection{Conclusions}

This paper demonstrates a novel CAE based approach to better retain and exploit engineering tacit knowledge. It presents an automated design framework for the development of individual part forming tools for a composite stiffener. The framework uses parametrically developed design geometries for both the part and its layup tool. The framework has been developed with a functioning user interface where part / tool combinations are developed using the interdependent dimensional relationships between the 'as designed' composite part and the tool required to form it. The tacit knowledge or 'know how' of the tooling engineer has also been coded into the tool covering design elements away from the part / tool interface. When complete the design options can be passed to a virtual environment for utility based assessment of their features and assemblability characteristics where again general tacit knowledge can be mobilized to create product specific knowledge based on practical build experience within the virtual environment. The work demonstrates clear benefits in automated process design methods with conventional design timelines reduced from hours and days to minutes and seconds. The methods developed here were able to produce a digital mockup of a component with its associated layup tool in less than 3 minutes. The methods presented here are best suited to the development of part families differing only in key dimensions or minor features. The time required to code the development of single, low value parts cannot be justified. The virtual environment presenting the design to the designer for interactive 
assembly planning was generated in 20 seconds and enabled the completion of virtual builds in support of the development of an optimal forming tool arrangement. This work discriminates itself from previous parametric design work through its extension of basic principles into the manufacturing domain. Through the virtual reality element it also provides a means by which generic tacit knowledge in assembly methods can be converted to product specific tacit knowledge through the completion of virtual builds. This then can be fed back to the parametric modelling system to improve the design in advance of production or any physical prototyping. This is important as not all knowledge required to develop an optimum system is available in advance of the design process when parametric methods are developed and applied. Challenges still exist in determining the level of reality required to provide an effective learning environment in the virtual world. The work also needs to be embedded in a real industrial environment to demonstrate and quantify benefits in a real industrial environment.

\subsection{References}

[1] Smith E. A. The Role of tacit and explicit knowledge in the workplace. Journal of Knowledge Management. 2001. Volume 5, Number 4, pp 311-321. Emerald Insight.

[2] Van Wijk R., Jansen J., Lyles M. Inter- and Intra-Organizational Knowledge Transfer: A Meta-Analytic Review and Assessment of its Antecedents and Consequences. Journal of Management Studies. June 2008. Volume 45, Issue 4, pp 830853. Wiley.

[3] Teece D.J. Strategies for Managing Knowledge Assets: the Role of Firm Structure and Industrial Context. Long Range Planning. 2000. Vol. 33, pp 35-54. Elsevier Science Ltd.

[4] Bellman, J. Knorr, A. Schomaker, R. Factors Influencing Time and Cost Overruns in Aircraft Projects. Aerlines Magazine. E-zine Edition, downloaded 31/1/16 49. pp 1 - 4. Aircraft Programs. https://aerlinesmagazine.files.wordpress.com/. Worldpress.

[5] Butler, A. Boeing's Cost Overruns Exceed \$1 Billion. Aviation Week Network. Aerospace Daily \& Defense Report. July 17 2015. Downloaded 31/1/16. http://aviationweek.com/. Downloaded 31/1/16. Aviation Week.

[6] Krishnakumar, K. Intelligent systems for aerospace engineering-An overview. NASA Technical Report, Document ID: 20030105746, 2003. downloaded August 2016, https://ti.arc.nasa.gov/m/pub-archive/364h/0364\%20(Krishna).pdf,

[7] Tong C., Duvvuru S. Artificial Intelligence in Engineering Design: Volume III: Knowledge Acquisition, Commercial Systems, And Integrated Environments. 20121.7 Summary pp 46-47. Elsevier.

[8] McMahon C., Lowe A., Culley S. Knowledge management in engineering design: personalization and codification. 2004 Journal of Engineering Design Volume 15 Issue 4, pp307-325. Taylor \& Francis.

[9] McConnell, R. Price, M. Butterfield J. Murphy A. Automated Methods for Aircraft Design Integrated with Manufacturing. The Royal Aeronautical Society's 4th Aircraft 
Structural Design Conference, $7^{\text {th }}-9^{\text {th }}$ October 2014, Queens University Belfast, United Kingdom.

[10] McConnell R., Butterfield J., Rafferty K., Price M., Raju B. \& Schulte B. Automated Methods for Virtual Product Development \& Aerospace Assembly Planning. International Manufacturing Conference IMC30, 3rd - 4th September 2013. University College Dublin, Ireland.

[11] Raymer D.P. Aircraft Design a Conceptual Approach. AIAA Education Series. American Institute of Aeronautics and Astronauts, 1992. ISBN 978-1-60086-911-2

[12] Pardessus T. The Multi-Site Extended Enterprise Concept in the Aeronautics Industry. Air and Space Europe May - August. 2001. Vol. 3, Issues 3 - 4, pp 46 - 48. Elsevier.

[13] Lam A. Tacit knowledge, organisational learning and innovation: A societal perspective. Danish Research Unit For Industrial Dynamics. DRUID Working Paper No. 98-22, 1998. Downloaded May 2016. http://www3.druid.dk. DRUID.

[14] Li S.T., Chang W.C.. Exploiting and Transferring Presentational Knowledge in R\&D Organisations. Expert Systems with Applications. 2009. Vol. 36, Issue 1, pp 766777. Elsevier.

[15] Ragan E.D., Huber K.J., Laha B., Bowman D.A. The Effects of Navigational Control and Environmental Detail on Learning in 3D Virtual Environments. Virtual Reality Workshops (VRW). 4 - 8 March 2012, Orange County California. IEEE.

[16] Senge P.M. The Fifth Discipline - The Art \& Practice of The Learning Organisation. 2nd. Edition. Page 26. Random House Business Books, London.

[17] Mujber T.S., Szecsi T., Hashmi M.S.J. Virtual Reality Applications in Manufacturing Process Simulation. Journal of Materials Processing Technology. 2004. Volumes 155-156, pp 1834-1838. Elsevier.

[18] Peng, Q., Chunsheng Y. Enhanced integrated manufacturing systems in an immersive virtual environment. Proceedings of the Institution of Mechanical Engineers, Part B: Journal of Engineering Manufacture. 2007. Vol. 221 No. 3, pp 477-487. SAGE Journals.

[19] Manesh H. F., Hashemipour M. Virtual-reality-based methodology for modelling and verifying shop floor control systems. Proceedings of the Institution of Mechanical Engineers, Part B: Journal of Engineering Manufacture. 2010. Vol. 224, No. 8. pp125165. Sage Journals.

[20] Korves B, Loftus M. The application of immersive virtual reality for layout planning of manufacturing cells. Proceedings of the Institution of Mechanical Engineers, Part B: Journal of Engineering Manufacture. 1999. Vol. 213, No. 1, pp 87-91. SAGE Journals.

[21] Pai Y., Yap H., Singh R. Augmented reality-based programming, planning and simulation of a robotic work cell. Proceedings of the Institution of Mechanical Engineers, Part B: Journal of Engineering Manufacture. 2015. Vol 229, No. 6. pp 102945. SAGE Journals. 
